\title{
Green beans (Phaseolus vulgaris, L.) quality loss upon thawing
}

\author{
R.C. Martins, C.L.M. Silva * \\ Escola Superior de Biotecnologia, Universidade Católica Portuguesa, Rua Dr. António Bernardino de Almeida, 4200-072 Porto, Portugal
}

Received 23 July 2003; accepted 8 December 2003

Keywords: Green beans; Thawing; Quality retention; Computer simulation

\begin{abstract}
Frozen green beans (Phaseolus vulgaris, L.) thawing is one of the operations that compromises significantly quality. The present research aims at studying the effects of thawing, at environmental and refrigeration temperatures, on the quality profile of a frozen green beans package. Quality losses were computationally evaluated, using a simulation system based on object-oriented technologies. Simulations show that sensory parameters, such as flavour and colour, are more sensitive to thawing at environmental temperatures, than nutritional parameters, such as vitamin $\mathrm{C}$ and starch contents.

The study demonstrates that green beans quality retention is also influenced by packaging materials, with different degrees of thermal insulation, and environmental conditions, such as temperature and surface heat convection coefficients.

Important conclusions are discussed on shelf-life limiting quality parameters during thawing and temperature abuses, as well as on thawing green vegetables to maximise their quality profile. Results emphasise that the principle of high-temperature-short-times is not directly applicable to frozen green beans thawing. Furthermore, simulations lead to the conclusion that overall quality profile is maximised by thawing under refrigeration temperatures.
\end{abstract}

\section{Introduction}

Green beans (Phaseolus vulgaris, L.) is one of the most important frozen vegetables consumed in Portugal. The outdoor varieties are produced seasonally and present high nutritional value (e.g. high content in water soluble vitamins, fiber and starch) and sensory attributes, such as colour, texture, aroma and flavour. Green beans contribute for a well-balanced diet, presenting nutritional advantages against human illness, which are common to green vegetables (Byers \& Perry, 1992).

Thawing properly is essential to maximise quality retention and safety of frozen vegetables. Thawing inside a refrigerator at temperatures below $5{ }^{\circ} \mathrm{C}\left(41^{\circ} \mathrm{F}\right)$, using a microwave oven or as part of the cooking process, are considered microbiologically safe methodologies. Microwave or simply direct cooking are recommended as part of any meal preparation. Thermal processing assures microbiological safety, when com-

\footnotetext{
* Corresponding author. Tel.: +351-22-5580058; fax: +351-225090351.

E-mail addresses: r.m.c.m@clix.pt (R.C. Martins), crislui@ esb.ucp.pt (C.L.M. Silva).
}

pared to thawing inside a refrigerator or at environmental temperatures. Thawing inside a refrigerator or at environmental temperature are important during prepreparation stages (e.g. frozen vegetables for salads and pies). Thawing overnight inside a refrigerator is practiced when processing and/or loss of soluble solids by water immersion thawing are not desired. Thawing is in fact a temperature abuse, and has to be considered a critical operation in terms of quality and safety.

Food safety has been addressed for the several thawing methods (Medeiros, Sanik, Miller, McCombs, \& Miller, 2001; Sage \& Ingham, 1998; Yamamoto \& Harris, 2001), whereas quality losses are not generally tackled by the scientific community. Nutritional losses, that occur during thawing, decrease the health benefits of frozen green vegetables included into a diet. These may also turn superfluous the efforts of temperature control throughout the frozen foods distribution chain, because quality can be severely affected at this final stage.

The time-temperature-tolerance project (TTT-project) did not covered thawing directly. Efforts were put primarily on frozen storage and temperature abuses effects on quality retention. These first studies showed 


\section{Nomenclature}

$\beta_{i} \quad$ quadratic polynomial constant

$\lambda$ thermal conductivity $\left(\mathrm{W} \mathrm{m}^{-1} \mathrm{~K}^{-1}\right)$

$\rho$ density $\left(\mathrm{kg} \mathrm{m}^{-3}\right)$

A $\quad T_{0}$, coded factor $\left({ }^{\circ} \mathrm{C}\right)$

AR autoregressive process

B $\quad T_{\infty}$, coded factor $\left({ }^{\circ} \mathrm{C}\right)$

c quality retention (\%)

C $\quad \lambda_{\mathrm{p}}$, coded factor $\left(\mathrm{W} \mathrm{m}^{-1} \mathrm{~K}^{-1}\right)$

$\mathrm{Cp}$ thermal capacity $\left(\mathrm{J} \mathrm{kg}^{-1} \mathrm{~K}^{-1}\right)$

D $\quad x_{\mathrm{p}}$, coded factor $(\mathrm{m})$

E $h$, coded factor $\left(\mathrm{W} \mathrm{m}^{-2} \mathrm{~K}^{-1}\right)$

FEM finite element method

$g \quad$ ratio of the force vector divided by $T_{0}$

$h$ surface heat transfer coefficient $\left(\mathrm{W} \mathrm{m}^{-2} \mathrm{~K}^{-1}\right)$

$k \quad$ kinetic rate $\left(\right.$ day $\left.^{-1}\right)$

$Y \quad$ response surface quality retention (\%)

ODE ordinary differential equation

\section{PDE partial differential equation \\ $T$ temperature $\left({ }^{\circ} \mathrm{C}\right)$ \\ $t \quad$ time (days) \\ TCD total colour difference \\ $x \quad$ packaging film thickness \\ AA ascorbic acid \\ DHAA dehydro-ascorbic acid}

\section{Subscripts}

$0 \quad$ refers to initial condition

$\infty \quad$ refers to environmental/refrigerator

air refers to air

eff refers to effective

eq refers to equilibrium

$\mathrm{H}$ refers to Hunter colour space

$\mathrm{p} \quad$ refers to package material

tray refers to tray that quality parameters of frozen vegetables, such as colour, flavour, texture, vitamin $\mathrm{C}$ and reducing sugars content are affected by temperature abuses (Dietrich, Bohart, Neumann, \& Morris, 1959, 1960, 1960).

More recently, isothermal and dynamic kinetic studies have shown that frozen storage affects significantly green beans quality, both in terms of nutritional and sensory parameters. Quality parameters, such as ascorbic acid (AA), total vitamin $\mathrm{C}$, starch and reducing sugars content, chlorophylls $a$ and $b$, colour and texture have shown to be very sensitive to frozen storage and temperature abuses (Martins \& Silva, 2002, 2003).

Computational simulation has also shown the sensitivity of green beans quality to frozen storage inside household refrigerators, under different storage temperatures, and also the impact of different packaging materials and storage conditions (Martins, 2004).

Ascorbic acid (AA) degradation through oxidation is common at low temperatures. AA is oxidised to dehydro-ascorbic acid (DHAA), which still retains vitamin C activity. Total vitamin $\mathrm{C}$ content of green beans is given by the sum of AA and DHAA contents. Vitamin C is lost when DHAA is oxidised to 2,3-diketo-L-gulonic acid. AA and DHAA are considered good indicators of water soluble vitamins of frozen vegetables, because other vitamins are generally more stable than vitamin C (Favell, 1998).

Green beans generally decrease its $\mathrm{pH}$ at low temperatures, because of their composition (Fennema, Powrie, \& Marth, 1973). The increase in $\mathrm{H}^{+}$concentration accelerates acid-catalysed reactions that affect colour, texture and flavour. The hydrolysis of structural sugars, such as pectins, the downshift in weight of hemi- celluloses and starch hydrolysis are responsible for the softening process at low temperatures. Furthermore, starch hydrolysis increases the free glucose content, increasing the sweetness intensity.

Colour loss at high storage temperatures (e.g. $T>-12{ }^{\circ} \mathrm{C}$ ) has been related to chlorophyll's pheophytisation. Pheophytisation is enhanced by the increase in $\mathrm{H}^{+}$concentration, in which the center magnesium of chlorophylls is replaced by hydrogen, forming pheophytine. Pheophytine gives an olive-brown colour to green beans. Colour is, nevertheless, stable at low temperatures (e.g. $T<-18{ }^{\circ} \mathrm{C}$ ). A possible explanation is the increase in concentration of metal ions, such as copper and zinc, which compete with $\mathrm{H}^{+}$for the center magnesium, forming metal-chlorophyll compounds that retain a vivid bright green colour.

Sensory properties are generally used to quantify frozen vegetables shelf-life. Properties, such as the perception of colour, texture and flavour, have been used to quantify quality, as perceived by the consumer. These have been considered shelf-life limiting factors, opposing to the nutritional and safety parameters in frozen vegetables. Nevertheless, sensory parameters may not always be the shelf-life limiting factors.

Quality losses during thawing are difficult to evaluate by experimental analysis. The different conditions, that affect heat transfer with phase and quality loss mechanisms, are not possible to be totally controlled. Quality parameters are also very diverse, and a large number of experimental conditions and replications have to be performed. Experimental limitations make most quality loss studies to neglect important factor interactions. Thus, it is difficult to transpose simple cause-effect explanations. 
The main motivation of this research is to evaluate frozen green beans quality profile during thawing at environmental and refrigeration temperatures, to produce detailed information on how quality retention decreases during thawing. Therefore, simulations were set to

I. Evaluate the impact of different packaging materials on frozen green beans quality retention during thawing.

II. Determine the shelf-life limiting quality parameters.

III. Derive conclusions on how to maximise quality retention upon thawing.

\section{Materials and methods}

\subsection{Computational model}

A simulation system was developed to model frozen foods quality loss. The system uses object-oriented scenario modelling techniques (Beringer, 1997) to set up models, such as the one presented in this research (Martins, 2004). The system promotes multi-physics capabilities, such as heat transfer and quality loss simulation. Scenario and object-oriented technologies allowed to develop distribution chain objects, such as the time-temperature spectrum simulator of room temperature and refrigerator cycles. The final objective of the simulation system was to provide a work base to obtain detailed information on food security, quality and shelflife dating tests.

Parallel/distributed programs were written in $\mathrm{C}++$ (Barton \& Nackman, 1991; Meyers, 1993, 1998) and let to run on a four node LINUX Beowulf Class Cluster (Radajewski \& Eadline, 1998a, 1998b; Sterling, Bell, \& Kowalik, 2001), under the parallel virtual machine (PVM) library (Geist et al., 1994). Systems philosophy is described in detail elsewhere (Martins, 2004).

Models use the finite elements method to discretise any PDE (partial differential equation) or ODE (ordinary differential equation) throughout the physical domain. The green beans package mesh, composed by a $900 \mathrm{~g}$ green beans package (bulk size: $198.14 \mathrm{~mm}$ width per $42.13 \mathrm{~mm}$ height $)$ on a polyethylene tray $(229.08 \mathrm{~mm}$ width, $11.92 \mathrm{~mm}$ height), under study is presented in Fig. 1.

Phase transition was modelled using a modified enthalpy formulation, whereby enthalpy is expressed as function of the apparent heat capacity (Martins, 2004).

Green beans thermal capacity and conductivity are described by a linear increase model with temperature above the freezing point. The apparent heat capacity and thermal conductivity below the freezing point were modelled by the modified Schwartzberg equation given in Ramaswamy and Tung (1981) and Schwartzberg (1976), respectively.

Quality losses kinetics are simulated by the first order and fractional conversion models in the ODE format, exhibiting Arrhenius behaviour with temperature (see Table 1). Kinetics were discretised into the green beans mesh by the variational method, using the consistent formulation and applied stochastically at nodal positions (Martins, 2004). The average quality retention through the bulk was computed for data analysis.

Air and tray thermal properties are considered constant during simulation $\left(\rho_{\text {air }}=1.18 \mathrm{~kg} \mathrm{~m}^{-3} ; \mathrm{Cp}_{\text {air }}=1004\right.$ $\mathrm{J} \mathrm{kg}^{-1} \mathrm{~K}^{-1} ; k_{\text {air }}=0.08 \mathrm{~W} \mathrm{~m}^{-1} \mathrm{~K}^{-1} ; \rho_{\text {tray }}=920 \mathrm{~kg} \mathrm{~m}^{-3}$; $\mathrm{Cp}_{\text {tray }}=2092 \mathrm{~J} \mathrm{~kg}^{-1} \mathrm{~K}^{-1} ; k_{\text {tray }}=0.33 \mathrm{~W} \mathrm{~m}^{-1} \mathrm{~K}^{-1}$ ).

Air voids inside package are considered trapped air, where heat transfer occurs by conduction. Heat conduction between green beans is considered minimal, as the contact area between green beans is reduced when compared to the surface area exposed to air voids (see Fig. 1).

Environmental temperature is simulated by an autoregressive process (AR) (Gardiner, 1997; Pandit \& Wu, 1983; Priestley, 1992). AR processes have been used to model temperature fluctuations inside refrigerated warehouses (Nicolai, 1994). Autoregressive model constants were obtained by regression against experimental time-temperature data, to reproduce the stochastic fluctuations of room temperature (Martins, 2004).

Refrigerator temperature cycles were modelled by a stable limit cycle (Tong, 1994). Temperature is described by a well defined periodic oscillation, fluctuating around a central area on a phase diagram. This oscillation is described by two discontinuous phases, or two different AR models, one for each compressor on/off cycles,

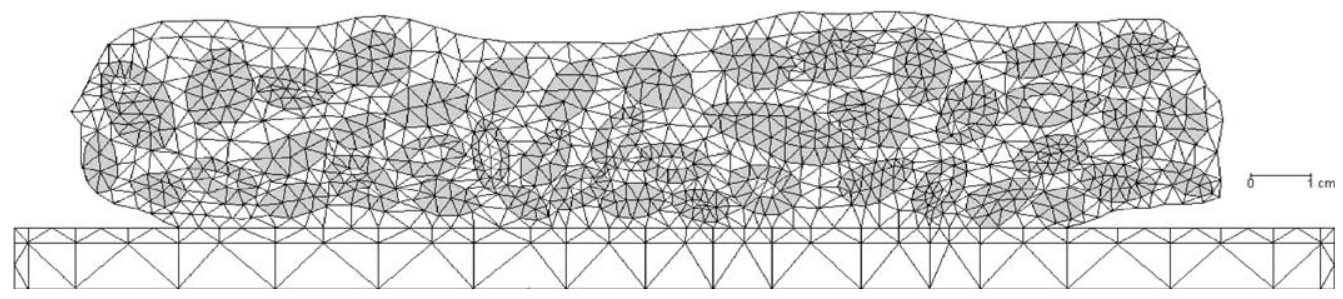

Fig. 1. Frozen green beans package FEM mesh on the polyethylene tray. 
Table 1

Green beans quality loss kinetic parameters

\begin{tabular}{|c|c|c|c|c|}
\hline Quality parameter & $C_{0}$ & $C_{\text {eq }}$ & $k_{\text {ref }}{ }^{\mathrm{a}}\left(\mathrm{day}^{-1}\right)$ & $E_{\mathrm{a}}\left(\mathrm{kJ} \mathrm{mol}^{-1}\right)$ \\
\hline Vitamin C (mg 100g $\left.\mathrm{g}^{-1}\right)$ & 146.1 & - & $3.226 \times 10^{-2}$ & 42.01 \\
\hline Starch $\left({\left.\mathrm{g} 100 \mathrm{~g}^{-1}\right)}\right.$ & 8.211 & - & $9.896 \times 10^{-2}$ & 12.33 \\
\hline Chlorophyll $a\left(\mathrm{mg} \mathrm{g}^{-1}\right)$ & $4.559 \times 10^{-2}$ & - & $4.793 \times 10^{-2}$ & 48.73 \\
\hline Chlorophyll $b\left(\mathrm{mg} \mathrm{g}^{-1}\right)$ & $2.572 \times 10^{-2}$ & - & $4.795 \times 10^{-2}$ & 49.60 \\
\hline$a$-Value & -11.931 & -6.329 & $6.600 \times 10^{-3}$ & 103.05 \\
\hline$b$-Value & 13.099 & 10.101 & $22.189 \times 10^{-3}$ & 55.33 \\
\hline $\mathrm{TCD}_{\mathrm{H}}$ & 3.745 & 7.486 & $5.999 \times 10^{-3}$ & 106.27 \\
\hline Stress (MPa) & 2.453 & 1.237 & $3.828 \times 10^{-2}$ & 7.11 \\
\hline Flavour ${ }^{\mathrm{b}}(\%)$ & 100 & - & $7.769 \times 10^{-2}$ & 117.00 \\
\hline
\end{tabular}

${ }^{\mathrm{a}} T_{\text {ref }}=-15^{\circ} \mathrm{C}$.

${ }^{\mathrm{b}}$ Data from Labuza (1982).

controlled by the refrigerator thermostat (Martins, 2004).

The developed model calculates the quality profile of green beans parameters: (i) ascorbic acid (AA); (ii) total vitamin $\mathrm{C}$ (ascorbic and dehydro-ascorbic acid contents); (iii) chlorophylls a and $b$; (iv) colour: Hunter $a$ and $b$ values and total colour difference, $\mathrm{TCD}_{\mathrm{H}}$; (v) texture: stress at the failure point; (vi) starch content (Martins, 2004); and (vii) flavour (data from Labuza, 1982). Table 1 presents the corresponding quality loss kinetic parameters.

Simulations were set to evaluate the impact on quality during $12 \mathrm{~h}$ at the environmental temperature $\left(+15{ }^{\circ} \mathrm{C}<T<+25^{\circ} \mathrm{C}\right)$ and $24 \mathrm{~h}$ at refrigeration temperatures $\left(+3{ }^{\circ} \mathrm{C}<T<+7{ }^{\circ} \mathrm{C}\right)$.

\subsection{Response surface methodology}

Quality losses along thawing were studied as a function of (i) green beans initial temperature, $T_{0}\left({ }^{\circ} \mathrm{C}\right)$; (ii) medium temperature, $T_{\infty}\left({ }^{\circ} \mathrm{C}\right)$; (iii) packaging thermal conductivity, $\lambda_{\mathrm{p}}\left(\mathrm{W} \mathrm{m}^{-1} \mathrm{~K}^{-1}\right)$; (iv) packaging film thickness, $x_{\mathrm{p}}(\mathrm{m})$; (v) time, $t$ (days); and (vi) surface heat transfer coefficient, $h\left(\mathrm{~W} \mathrm{~m}^{-2} \mathrm{~K}^{-1}\right)$.

Experimental design maximal and minimal levels are presented in Table 2 for thawing at environmental and refrigeration temperatures. Factor combinations were designed using the Box-Behnken design (Montegomery, 1991). The design totallised 46 combinations organised in one block. Quality retention (\%), $Y$, is assumed to be described by a second order polynomial:

$$
\begin{aligned}
Y= & \beta_{0}+\beta_{1} \mathbf{A}+\beta_{2} \mathbf{B}+\beta_{3} \mathbf{C}+\beta_{4} \mathbf{D}+\beta_{5} \mathbf{E}+\beta_{12} \mathbf{A} \mathbf{B} \\
& +\beta_{13} \mathbf{A C}+\beta_{14} \mathbf{A D}+\beta_{15} \mathbf{A E}+\beta_{23} \mathbf{B C}+\beta_{24} \mathbf{B D} \\
& +\beta_{25} \mathbf{B E}+\beta_{34} \mathbf{C D}+\beta_{35} \mathbf{C E}+\beta_{45} \mathbf{D E}+\beta_{11} \mathbf{A}^{2} \\
& +\beta_{22} \mathbf{B}^{2}+\beta_{33} \mathbf{C}^{2}+\beta_{44} \mathbf{D}^{2}+\beta_{55} \mathbf{E}^{2}
\end{aligned}
$$

where $\mathbf{A}, \mathbf{B}, \mathbf{C}, \mathbf{D}$ and $\mathbf{E}$ are the coded factors for $T_{0}, T_{\infty}$, $\lambda_{\mathrm{p}}, x_{\mathrm{p}}$, and $h$, respectively. In this design, only secondorder interactions were considered in the surface analysis.

The parameters $\beta_{i}$ of Eq. (1) were determined using the software Design Expert (version 4.0.2) (Stat-Ease, 1994). Residuals adequacy were analysed for outlayers, randomness and tested for normality (Bates \& Watts, 1988; Box, Hunter, \& Hunter, 1978). Factors statistical significance and their interactions were computed by the studentised effect $\left(\beta_{i} / s\left\{\beta_{i}\right\}\right)$, at a $5 \%$ confidence level (double sided $T$-test, $t_{0.975 ; 25}=2.060$ ) (Neter, Kutner, Nachtsheine, \& Wasserman, 1996). Model prediction adequacy and fit was accessed by the ANOVA, one factor $(\alpha=0.05)$, without replication. ANOVA (one factor, $\alpha=0.05$ ) was also used to determine significant differences between quality retentions.

Response surface regressions were obtained for vitamin $\mathrm{C}$ and flavour retentions, during thawing at environmental and refrigeration temperatures. These

Table 2

Factor and factor levels used on the design of thawing simulations

\begin{tabular}{lllll}
\hline \multirow{2}{*}{ Factor } & \multicolumn{2}{l}{ Environmental temperature } & & \multicolumn{2}{l}{ Refrigeration temperature } \\
\cline { 2 - 5 } & Min & Max & Min & Max \\
\hline A: Initial temperature $\left({ }^{\circ} \mathrm{C}\right)$ & -30 & -5 & -30 & -5 \\
B: Environmental temperature $\left({ }^{\circ} \mathrm{C}\right)$ & +15 & +25 & +37 & +7 \\
C: Thermal conductivity $\left(\mathrm{W} \mathrm{m}^{-1} \mathrm{~K}^{-1}\right)$ & 0.02 & 0.15 & 0.02 & 0.15 \\
D: Packaging film thickness $(\mathrm{m})$ & 0.0002 & 0.003 & 0.002 & 0.003 \\
E: Surface heat transfer coefficient & 6 & 12 & 6 & 12 \\
$\quad\left(\mathrm{~W} \mathrm{~m}^{-2} \mathrm{~K}^{-1}\right)$ & & &
\end{tabular}


parameters were selected, because represent nutritional and sensory properties, and extreme behaviour in terms of kinetics sensitivity to temperature (vitamin $\mathrm{C}$ with low $E_{\mathrm{a}}$ and flavour with high $E_{\mathrm{a}}$ ). Furthermore, these were repeated for the heuristical sampling times of (i) 3, 6,9 , and $12 \mathrm{~h}$ for thawing at environmental temperatures; and (ii) $6,12,18$ and $24 \mathrm{~h}$ for thawing at refrigeration temperatures.

\subsection{Determination of quality retention at the thawing time}

The thawing time of a green beans package was considered to be the time elapsed between time zero and the time when the last node attains the melting point temperature, $T_{\mathrm{m}}=-0.2{ }^{\circ} \mathrm{C}$ (Martins, 2004). Thereafter, the quality profile at this time is recorded, and the average, maximum and minimum values are recorded. These simulations were carried for the same conditions described in Section 2.2.
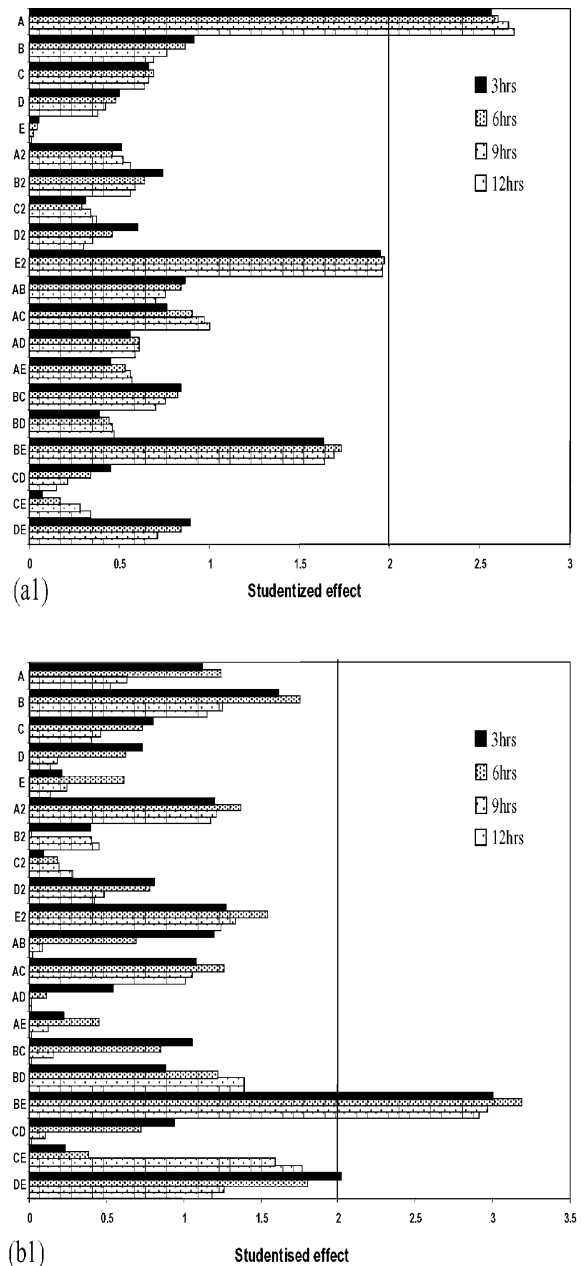

\subsection{Quality profile analysis}

The quality profile was registered at heuristical time intervals. The average, maximum and minimum quality retentions were obtained at (i) 3, 6, 9, and $12 \mathrm{~h}$ for thawing at environmental temperatures, and (ii) 6, 12, 18 and $24 \mathrm{~h}$ for thawing at refrigeration temperatures. The calculations were also carried out for all the same conditions in Section 2.2.

\section{Results and discussion}

\subsection{Variable effects on quality retention}

Fig. 2 presents the studentised effect ( $t$-ratio, double sided $t$-test) of the response surface coefficients $\beta_{i}$, for vitamin $\mathrm{C}$ and flavour retention, $t$-values above the $t$ critical line are interpreted as significant response surface coefficients.
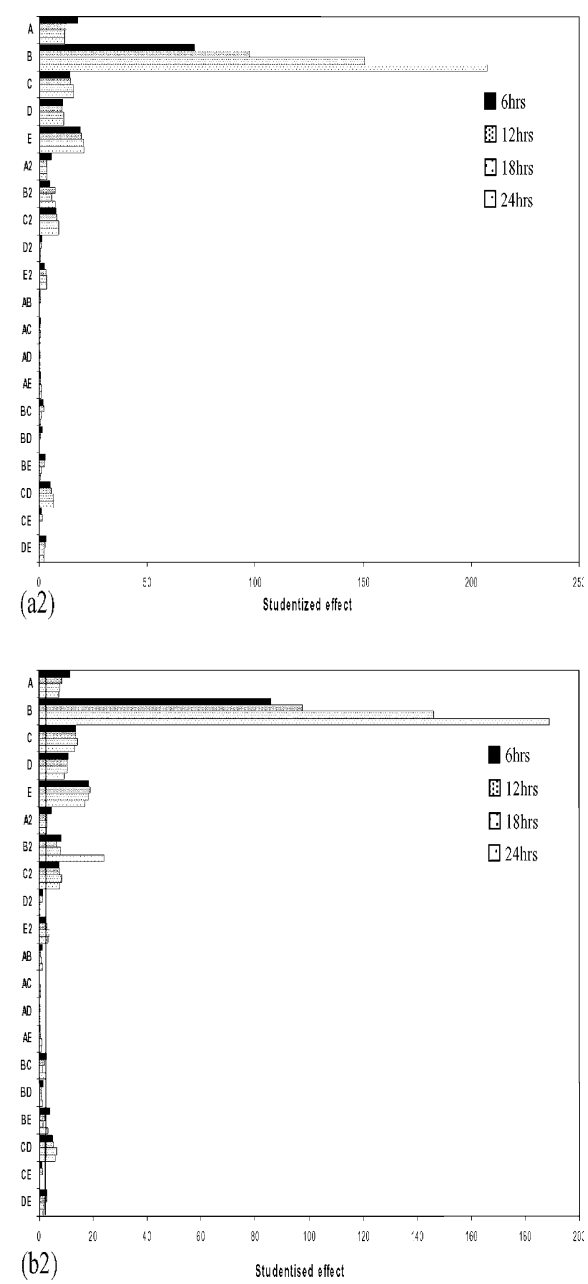

Fig. 2. Studentised effects of response surface coefficients for quality retention upon thawing: (a1) vitamin $\mathrm{C}$ at environmental temperature; (a2) vitamin C inside refrigerator; (b1) flavour retention at environmental temperature; (b2) flavour retention inside the refrigerator; $\left(t_{\alpha=0.975: 25}=2.060\right)$. 
Thawing is a highly non-linear phenomena, and this is expressed on the lack of a smooth pattern on the quality loss response surfaces. Fig. 2 (a2) and (b2) show that the studied variables (A, B, C, D and E) are significant on flavour and vitamin $\mathrm{C}$ retentions, as well as their interactions during thawing inside the refrigerator. At the environmental temperature, the initial temperature (A) and heat surface convection coefficient (E) are the most significant factors on vitamin $\mathrm{C}$ retention (Fig. 2 (a1)), and the interaction between the medium temperature (B) and heat surface convection coefficient (E) is the most significant factor on flavour retention (Fig. 2(b1)).

Quality retention is dependent upon the internal mechanisms of heat transfer, phase transition and quality degradation kinetics, as well as the medium conditions that affect heat transfer. Thawing presents both a structural and a dynamic complexity, that is difficult to be analysed just by cause-effect relationships.

Quality loss variance shows not to be a direct cause of single factors, but, rather, is obtained by the combinations of $T_{0}, T_{\infty}, \lambda_{\mathrm{p}}, x_{\mathrm{p}}$ and $h$. Combinations that enhance low thermal insulation, high medium temperatures and initial temperatures lead to lower quality retention for all the studied quality parameters. High thermal insu- lation materials, low $T_{\infty}$ and low $T_{0}$ enhance better quality retention patterns.

The initial temperature has a small effect on the total thawing time inside the refrigerator, and consequently on quality retention. The use of low initial temperatures for thawing at environmental temperatures produces significant increases in the thawing time, enhancing better quality retentions. At low temperatures, green beans exhibit a small thermal capacity and high thermal conductivity, which makes temperature to increase very rapidly until near the melting point temperature. On the other hand, large temperature gradients, inside the green beans package, contribute also for the rapid increase of temperature during thawing. This can be observed in Fig. 3 .

Furthermore, high medium temperatures decrease significantly the thawing time (see Fig. 3(a1) and (a2)) whereas, refrigeration temperatures near by the melting point are able to maintain the green beans package frozen for longer periods (see Fig. 3(b1) and (b2)). Such differences on the thawing time can significantly affect the quality of frozen vegetables. Such analysis will be addressed in Section 3.2.

The effect of thermal insulation and convection at the package surface is easier to be analysed by an effective surface heat transfer convection coefficient, $h_{\text {eff }}$ :
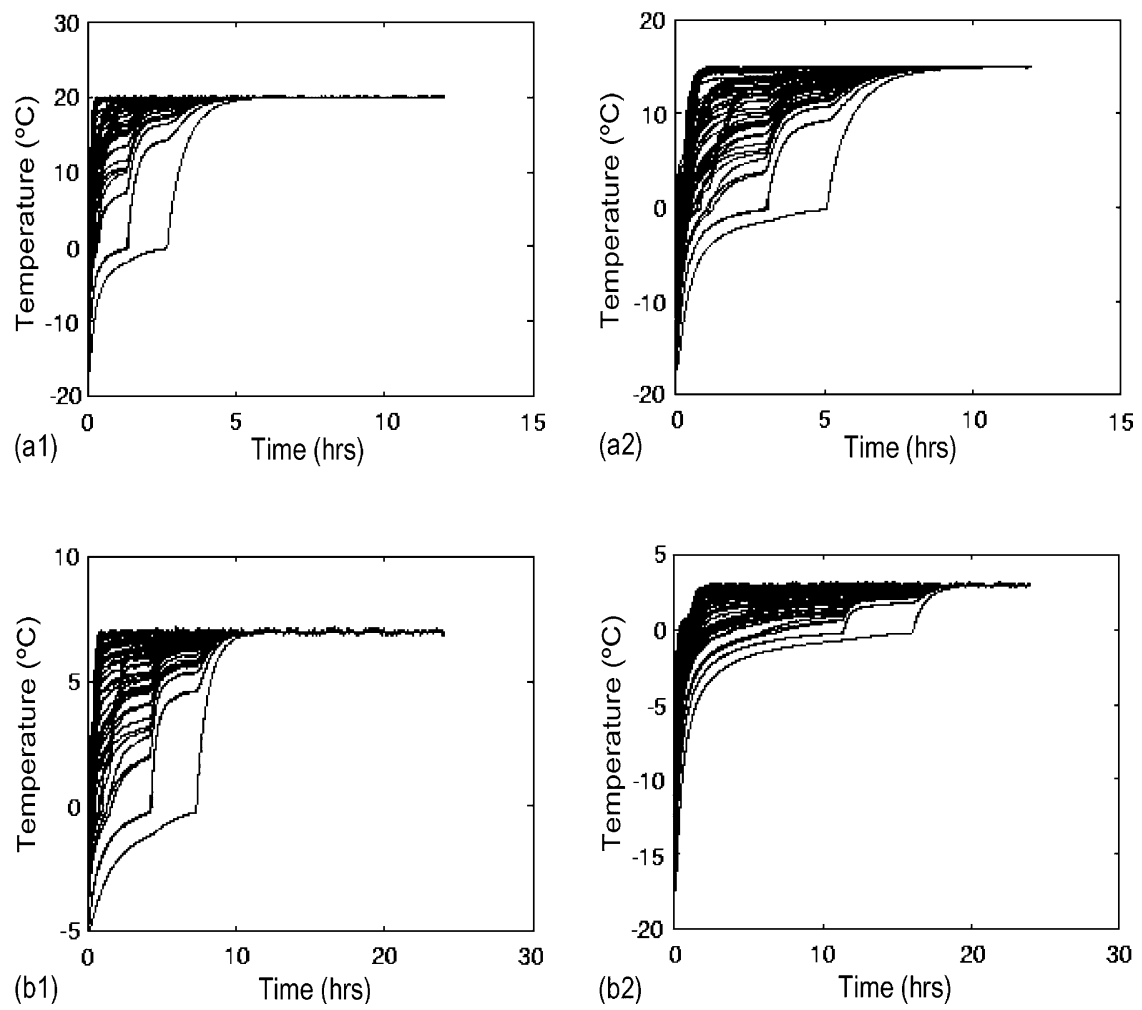

Fig. 3. Time-temperature spectrum inside the green beans package at selected nodal points, for the maximum and minimum thawing times recorded at environmental and refrigeration temperatures. (a1) Minimum thawing time case at environmental temperature; (a2) maximum thawing time case at environmental temperature; (b1) minimum thawing time case inside the refrigerator; and (b2) maximum thawing time case inside the refrigerator. 
$h_{\mathrm{eff}}=\left(\frac{1}{h}+\frac{x_{\mathrm{p}}}{k_{\mathrm{p}}}\right)^{-1}$

where $h$ is the surface convection coefficient $\left(\mathrm{W} \mathrm{m}^{-2} \mathrm{~K}^{-1}\right), \lambda_{\mathrm{p}}$ the packaging material thermal conductivity $\left(\mathrm{W} \mathrm{m}^{-1} \mathrm{~K}^{-1}\right)$ and $x_{\mathrm{p}}$ the package thickness $(\mathrm{m})$. Combinations of low $h, \lambda_{\mathrm{p}}$ and high $x_{\mathrm{p}}$ values lead to small effective surface coefficients, $h_{\text {eff }}$. This makes these variables extremely dependent, for which the independent effects are not as significant as their different combinations.

To illustrate the combined impact of $T_{0}, T_{\infty}, \lambda_{\mathrm{p}}, x_{\mathrm{p}}$ and $h$, the following factor is suggested:

$g=\frac{T_{\infty} \cdot h_{\text {eff }}}{T_{0}}$

which is the ratio of the force vector, $T_{\infty} \cdot h_{\mathrm{eff}}$, by the initial temperature, $T_{0}$.

Fig. 4 presents the effect of $g$ on vitamin $\mathrm{C}$ and flavour retentions during thawing at environmental and refrigeration temperatures. The influence of $g$ on the quality is more significant at refrigeration temperatures, than at environmental temperatures (see Fig. 4). Furthermore, the $g$ factor has more impact on flavour degradation than on vitamin $\mathrm{C}$ retention. This was expected, since flavour degradation kinetics is more sensitive to temperature (larger $E_{\mathrm{a}}$, see Table 1).

Temperature differences between the green beans package and the medium temperature, during thawing at room temperature, are responsible for the small effect of $g$ on quality retention (Fig. 4(a1) and (b1)). Such result emphasises that the packaging materials $\left(x_{\mathrm{p}}\right.$ and $\lambda_{\mathrm{p}}$ ) do not make a significant thermal insulation against environmental temperatures, within the range of $T_{\infty}+15^{\circ} \mathrm{C}$ to $+25^{\circ} \mathrm{C}$. Under such conditions, the only protection against quality losses is the latent heat of fusion.

Quality retention is highly affected by the medium temperature. Green beans thawed inside the refrigerator exhibit higher quality retention, over the quality profile, after $24 \mathrm{~h}$ than when thawed at the environmental temperature for a period of $12 \mathrm{~h}$ (see Section 3.3).

Temperature differences between the green beans package and the temperature inside the refrigerator (Fig. 4(a2) and (b2)) are not as significant as when thawing at the environmental temperature. Thus, the packaging materials properties $\left(x_{\mathrm{p}}\right.$ and $\left.\lambda_{\mathrm{p}}\right)$ are, at these temperatures, capable of significant thermal insulation.

Thawing inside the refrigerator maximises the thawing time. Maintaining temperatures near the melting
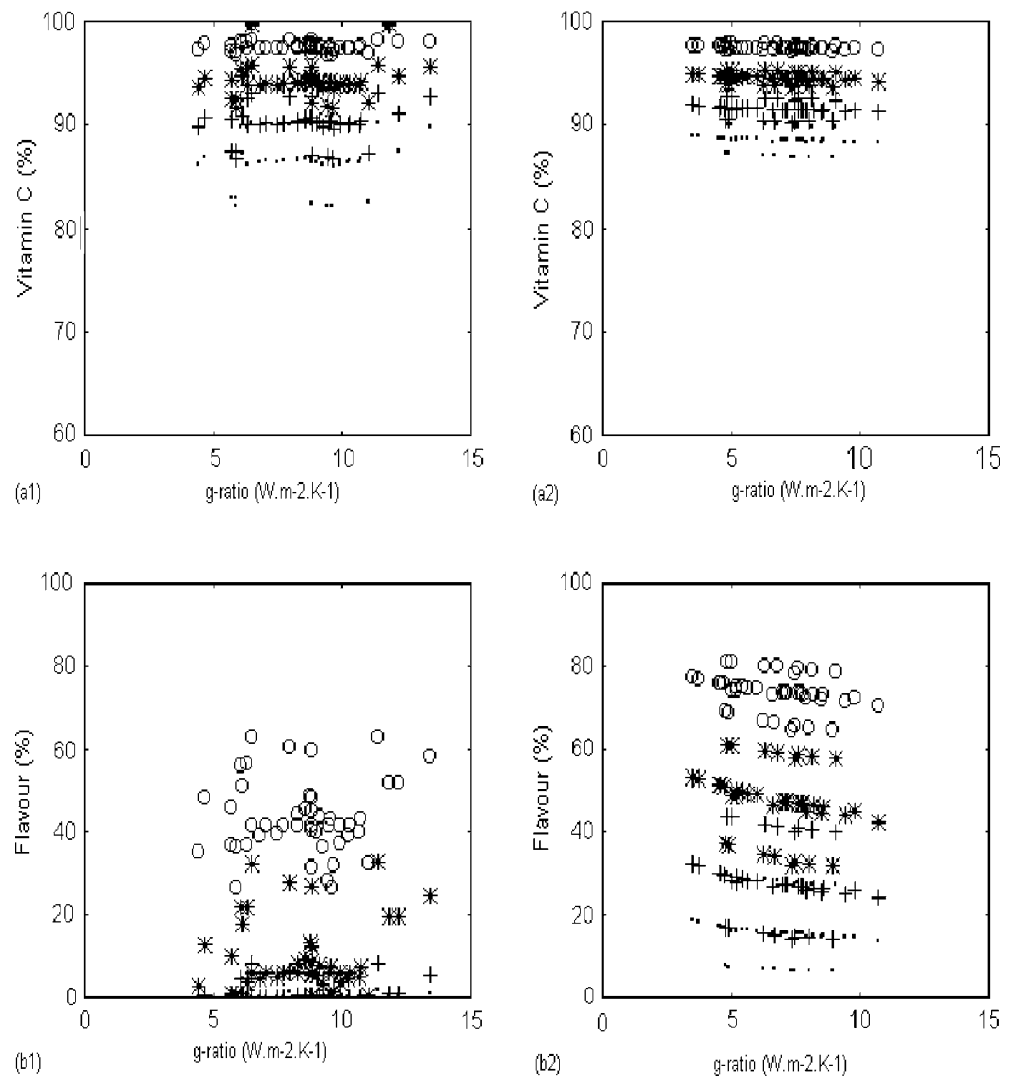

Fig. 4. Green beans vitamin $\mathrm{C}$ and flavour retentions as function of the $g$-ratio. (a1) Vitamin $\mathrm{C}$ at the environmental temperature, where $\mathrm{O}, *,+$, and $\cdots$ are sampled at 3, 6, 9, and $12 \mathrm{~h} ;(\mathrm{a} 2)$ vitamin $\mathrm{C}$ at the refrigeration temperature, where $\mathrm{O}, *$, + , and $\cdots$ are sampled at $6,12,18$, and $24 \mathrm{~h}$; (b1) flavour at the environmental temperature, where $\mathrm{O}, *,+$, and $\cdots$ are sampled at 3,6, 9, and $12 \mathrm{~h}$; (b2) flavour at the refrigeration temperature, where $\mathrm{O}, *,+$, and $\cdots$ are sampled at $6,12,18$, and $24 \mathrm{~h}$. 
point, increases frozen green beans quality retention during thawing.

\subsection{Quality retention at thawing time}

Thawing curves are presented in Fig. 3. The average thawing time at the slowest thawing point, at medium temperature, is $219 \mathrm{~min}(3.65 \mathrm{~h})$, with maximal and minimal values of $306 \mathrm{~min}$ and $160 \mathrm{~min}(5.1$ and $2.67 \mathrm{~h}$ ), respectively. The thawing time range emphasises the significance of the initial and medium temperatures and thermal insulation. Maximum thawing times are obtained under lower initial and medium temperatures and packages with high thermal insulation, whereas the minimum thawing times occur under high $T_{0}$ and $T_{\infty}$, and low thermal insulation.

Similar differences are observed inside the refrigerator (see Fig. 3(b1) and (b2)). Average thawing times range $639.15 \mathrm{~min}(10.65 \mathrm{~h})$, with maximum and minimum values of $966 \mathrm{~min}$ and $439 \mathrm{~min}(16.1$ and $7.31 \mathrm{~h}$ ), respectively. As expected, the same combinations of factors produce maximum/minimum thawing times.

Quality loss profile at the thawing point is presented in Fig. 5. It is possible to observe that most quality parameters exhibit higher retentions when thawed at the environmental temperature than inside the refrigerator. Exceptions are shown for sensory attributes, such as colour, $a$-value and $\mathrm{TCD}_{\mathrm{H}}$, and flavour. These are the quality parameters with kinetics with higher sensitivity to temperature (higher $E_{\mathrm{a}}$ ).
Nutritional parameters are generally well retained until the melting point inside the refrigerator. Total vitamin $\mathrm{C}$ and starch are retained on average 95.36\% and $93.90 \%$, with low dispersion between maximum and minimum values.

Chlorophylls $a$ and $b$ are retained on average $91.77 \%$ and $91.48 \%$, respectively, with low dispersion.

Colour is generally well retained inside the refrigerator. The Hunter $b$-value presents non-significant degradation, attaining $98.91 \%$ at the thawing point, with very low dispersion. The Hunter $a$-value decreased on average to $97.22 \%$, and the total colour difference exhibits an increase of $5.93 \%$.

Texture is also well retained during thawing inside the refrigerator. Very high retentions are obtained, 99.28\%, with small dispersion.

Flavour is, however, not well retained until the thawing point. Average retention is $54.85 \%$, with maximum and minimum retentions of $65.28 \%$ and $46.09 \%$ respectively.

Nutritional parameters attain higher retentions when green beans are thawed at environmental temperatures. Total vitamin $\mathrm{C}$ and starch are retained on average $96.92 \%$ and $97.42 \%$, respectively.

Chlorophylls $a$ and $b$ are also well retained during thawing at environmental temperatures, where retentions are on average $93.78 \%$ and $93.41 \%$.

Lower retentions are obtained in terms of colour, when compared to thawing at refrigeration temperatures. The $a$-value is reduced on average to $93.76 \%$, with maximum and minimum values of $97.10 \%$ and $90.46 \%$,

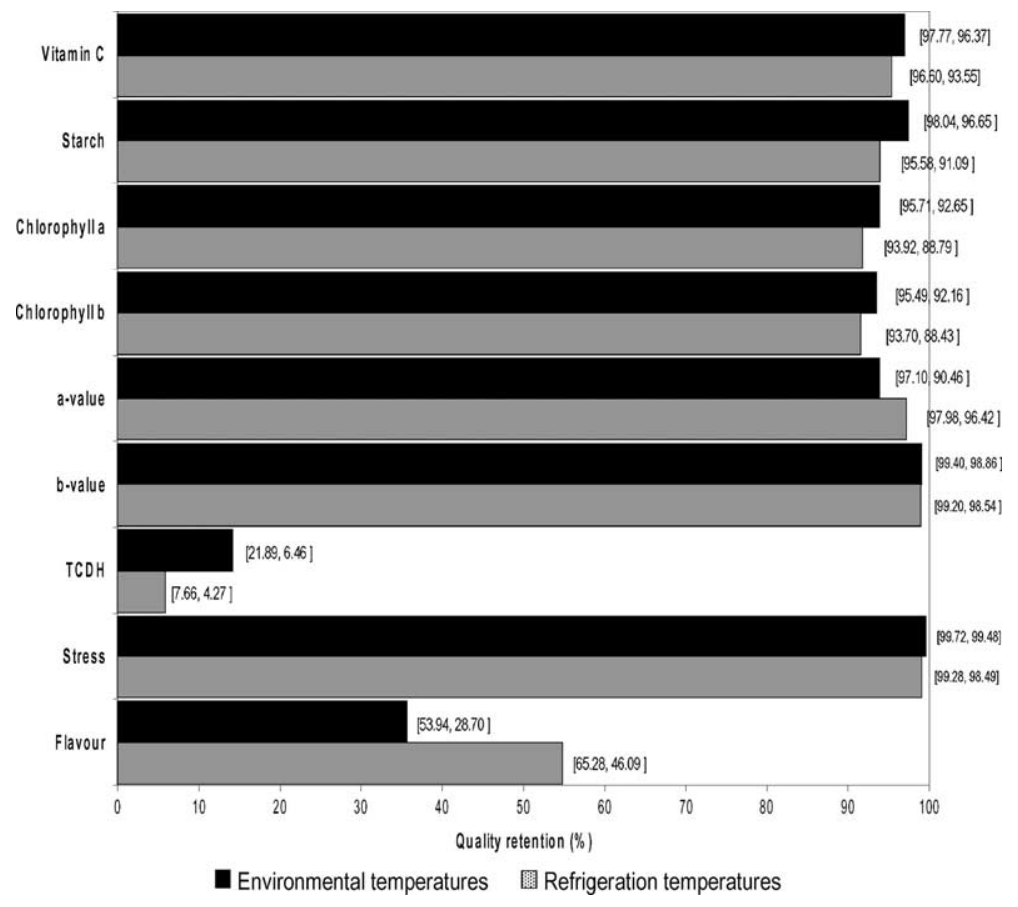

Fig. 5. Maximum and minimum average quality retentions at thawing time, under environmental and refrigeration temperatures. 
respectively. The Hunter $b$ coordinate is not severely affected, where average retention is $99.07 \%$. However, $\mathrm{TCD}_{\mathrm{H}}$ are significant when thawing at environmental temperatures. $\mathrm{TCH}_{\mathrm{H}}$ increases on average $14.19 \%$, and has a high dispersion of maximum-minimum results, $21.89-6.46 \%$.

Texture is not affected significantly during thawing at environmental temperatures (average retentions of $99.62 \%$, with very low dispersion).

Flavour retention is highly affected by thawing at environmental temperatures. Average retention is $35.63 \%$, with a large maximum-minimum dispersion, $53.94-28.70 \%$, respectively. Flavour retention is highly affected by thermal insulation and initial and environmental temperatures.

\subsection{Quality profile analysis}

Average quality retention profiles are presented in Figs. 6 and 7, for thawing at environmental and refrigeration temperatures, respectively. The comparison of these figures allows to conclude that the exposure at the environmental temperature during $12 \mathrm{~h}$, is generally more detrimental than the $24 \mathrm{~h}$ period inside the refrigerator.

Total vitamin $\mathrm{C}$ and starch contents are generally well retained throughout the $12 \mathrm{~h}$ of exposure at the environmental temperature, where, on average, retention attains the value of $86.28 \%$ and $91.01 \%$, respectively.

The low dispersion assures that vitamin $\mathrm{C}$ and starch contents are not severely affected by the different combinations of packaging materials and storage conditions.

Chlorophylls $a$ and $b$ losses are highly affected by the exposure to environmental temperatures, where average retentions are, respectively, $71.44 \%$ and $73.48 \%$. Chlorophyll retention is highly affected by storage conditions and packaging materials, where the combined effects reached the significant dispersion of $18.09 \%$ (16.91\%) and $24.2 \%(20.09 \%)$ for chlorophylls $a$ and $b$, respectively (see Fig. 6).

Colour is significantly affected by the chlorophylls degradation. The Hunter $a$-value decreases on average $73.48 \%$, and the $b$-value $95.67 \%$. The $\mathrm{TCD}_{\mathrm{H}}$ is highly affected by thawing at environmental temperatures. $\mathrm{TCD}_{\mathrm{H}}$ increases on average $59.11 \%$, with a very large dispersion between maximum and minimum retentions ( $84.56 \%$ and $31.45 \%$, respectively). Thus, colour retention is highly affected by the environmental temperature, thermal insulation provided by the packaging material, and surface heat transfer coefficient.

Texture is well retained during thawing at environmental temperatures. Stress at the failure point decreases only on average $98.67 \%$, with low dispersion

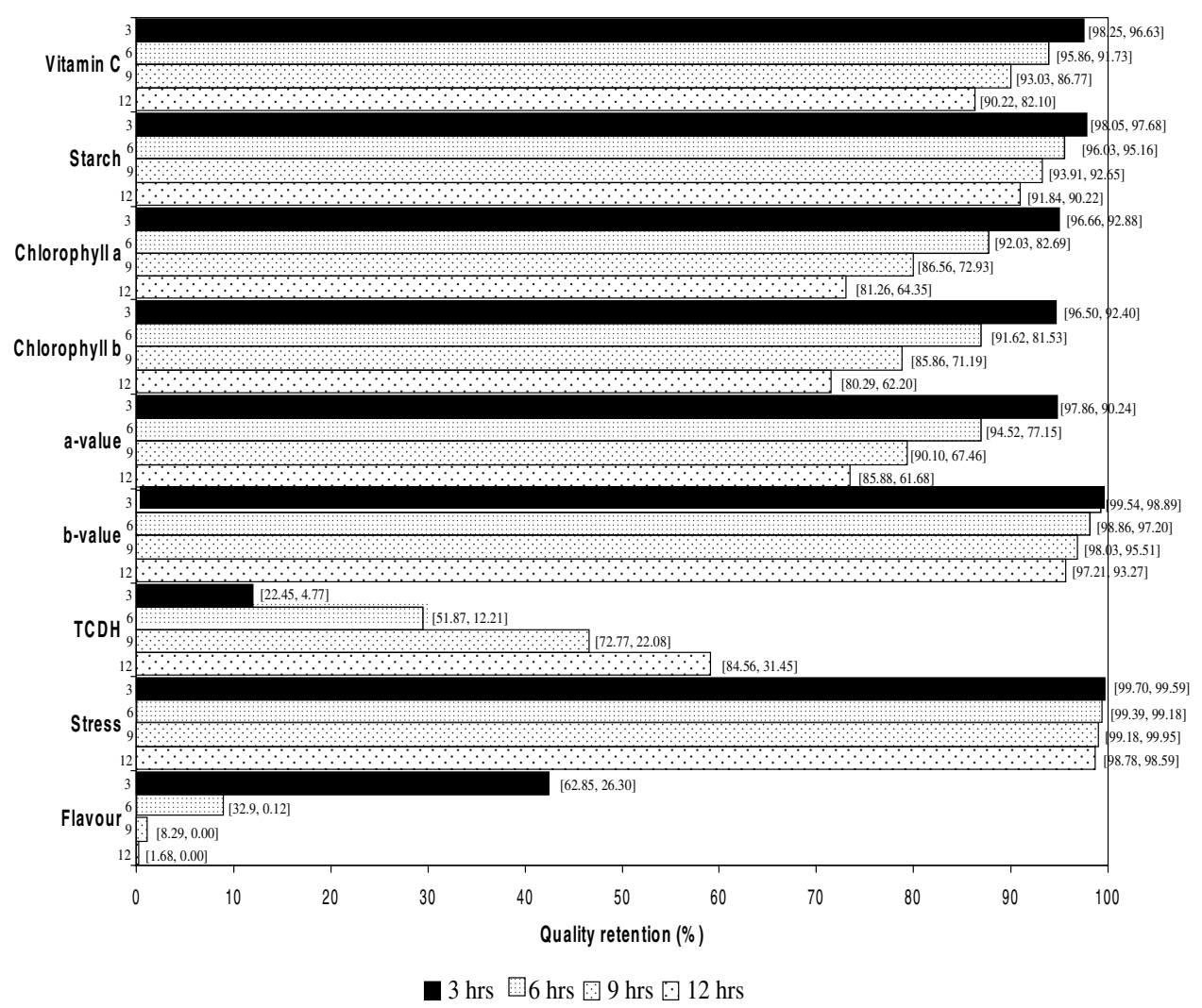

Fig. 6. Green beans average quality retention (\%) during thawing at the environmental temperature, at 3, 6, 8, and $12 \mathrm{~h}$. Maximum and minimum limits are presented within brackets. 
(see Fig. 6). Such result agrees with the low starch degradation during the thawing operation.

In the first $3 \mathrm{~h}$ of exposure, flavour is highly affected by thawing at environmental temperature. Flavour retention attains on average $42.46 \%$, with maximum and minimum retentions of $62.85 \%$ and $26.30 \%$.

Flavour retention constrains the quality of frozen green beans, that are thawed at the environmental temperature, where retentions are highly dependent on thermal insulation and environmental conditions. Thus, under a worst case scenario, of minimal thermal isolation, $50 \%$ of flavour retention is lost after $1 \mathrm{~h}$ of exposure to environmental conditions.

Thawing inside the refrigerator is less detrimental than thawing at environmental temperatures. Good vitamin $\mathrm{C}$ and starch retentions are achieved during a 24 $\mathrm{h}$ period at refrigeration temperatures, where average retentions range $88.54 \%$ and $86.35 \%$, respectively (Fig. 7).

Chlorophylls $a$ and $b$ are moderately affected by thawing inside the refrigerator. Average retentions of $79.91 \%$ and $79.20 \%$ are obtained for chlorophylls $a$ and $b$, respectively. Significant dispersion is obtained for this quality parameter, where maximum and minimum retentions of $82.87-76.79 \%$ and $82.33-75.90 \%$ are obtained for chlorophylls $a$ and $b$.
Colour is moderately retained during the $24 \mathrm{~h}$ period inside the refrigerator. At the end of this period, the Hunter $a$ and $b$ average values are $92.25 \%$ and $97.24 \%$.

$\mathrm{TCD}_{\mathrm{H}}$ exhibits an average increase of $16.63 \%$ on average, with maximum and minimum retentions of $22.69 \%$ and $11.48 \%$, respectively. The large variation emphasises that thermal isolation and refrigeration conditions significantly affect $\mathrm{TCD}_{\mathrm{H}}$.

Flavour is also affected during thawing inside the refrigerator. Average retentions attain values of $16.37 \%$ at the end of the $24 \mathrm{~h}$. Flavour losses are already very significant during the first $12 \mathrm{~h}(47.31 \%$, with $60.99 \%$ and $31.60 \%$ for maximum and minimum retentions, respectively).

\subsection{Effect of temperature}

The effect of storage temperature on quality loss kinetic rates is presented in Fig. 8. The negative slope of each curve represents the ratio of the Arrhenius activation energy $\left(E_{\mathrm{a}}\right)$ by the universal gas-constant $(R)$, presented in Table 1.

Quality loss parameters with higher activation energies are more sensitive to temperature, exhibiting a higher negative slope in Fig. 8. In this figure, it is pos-

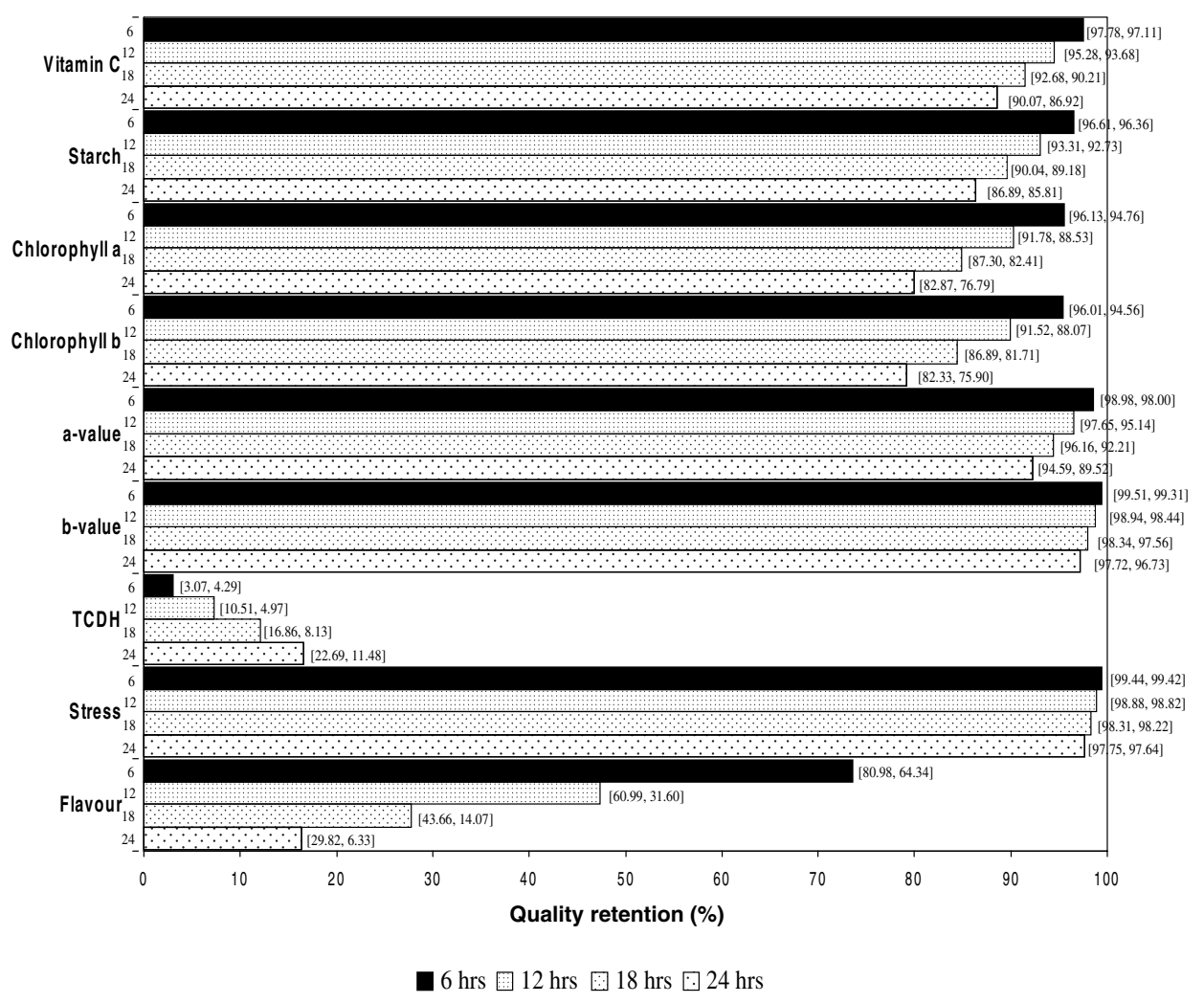

Fig. 7. Green beans quality retention (\%) during thawing inside the refrigerator at 6,12,18 and $24 \mathrm{~h}$. Maximum and minimum limits are presented within brackets. 


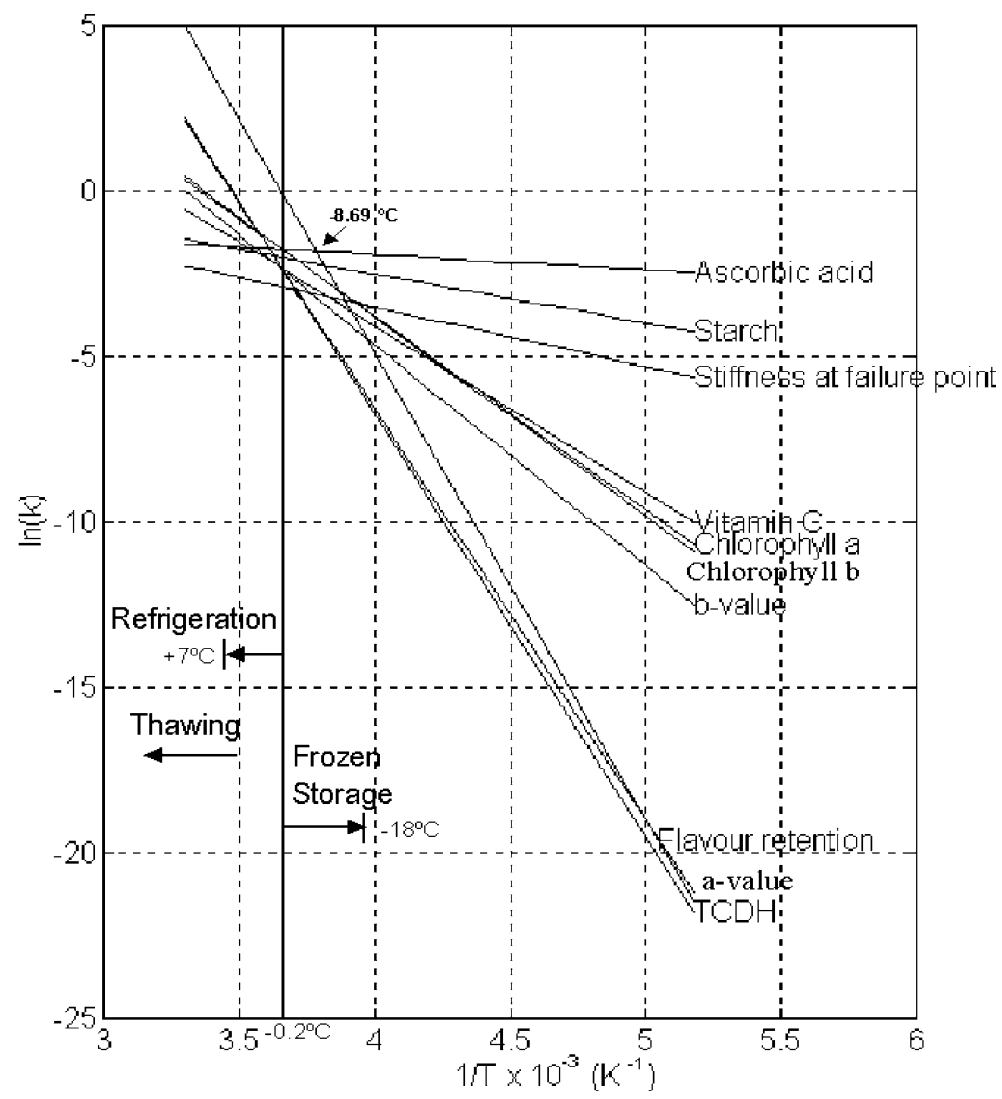

Fig. 8. Logarithmic plot of the kinetic rate as function of the inverse of the absolute temperature.

sible to identify three major groups of quality parameters:

I. Low $E_{\mathrm{a}}$, such as ascorbic acid auto-oxidation, starch hydrolysis and texture loss, such as stiffness and stress at failure point.

II. Intermediate $E_{\mathrm{a}}$, such as total vitamin $\mathrm{C}$ degradation (AA + DHAA oxidation), chlorophylls $a$ and $b$ losses, and the Hunter $b$-coordinate.

III. High $E_{\mathrm{a}}$, such as flavour retention and colour (total colour difference and Hunter $a$-value).

Sensory parameters present higher temperature sensitivity (higher $E_{\mathrm{a}}$ ). Low storage temperatures (e.g. $T<-18{ }^{\circ} \mathrm{C}$ ) are capable to reduce significantly quality losses of sensory parameters (e.g. flavour and colour), whereas, nutritional parameters are not significantly retained. Consequently, low storage temperatures are capable of maintaining colour and flavour for long storage periods, but not nutritional parameters, such as ascorbic acid and starch content. Texture is an exception to the sensory parameters group behaviour, because low temperatures are not capable of decreasing its degradation.

Storing green beans at refrigeration temperatures has the opposite effect (see Fig. 8). Under refrigeration conditions $\left(+3{ }^{\circ} \mathrm{C}<T<+7{ }^{\circ} \mathrm{C}\right)$, sensory parameters, such as flavour and colour, exhibit higher degradation rates than parameters such as ascorbic acid, starch and texture loss. At temperatures above the melting point, sensory parameters are therefore shelf-life limiting factors, being, however, this observation limited by the non-linearity of phase transition and heat transfer.

Thus, for most severe temperature abuses, such as thawing at environmental temperatures, it is expected that colour and flavour are highly affected, whereas vitamin $\mathrm{C}$ and starch contents are not affected to the same extent. This has been confirmed in Section 3.3.

Unfortunately, for shelf-life predictions, most curves intercept at refrigeration temperatures $\left(-18{ }^{\circ} \mathrm{C}<T<-7\right.$ ${ }^{\circ} \mathrm{C}$ ) (see Fig. 8). It is possible to observe that ascorbic acid constrains the quality of frozen green beans at storage temperatures below $-8.69{ }^{\circ} \mathrm{C}$. Above this temperature, flavour degradation is the most sensitive quality parameter.

Important considerations can be made from the frozen green beans quality loss global behaviour. Thawing at high temperatures maximises nutritional retention (e.g. ascorbic acid), whereas, thawing inside the refrigerator is capable of maximising sensory attributes (e.g. colour and flavour). However, by thawing inside the refrigerator at low temperatures (e.g. near the melting point of green beans) it is possible to obtain a balance between sensory and nutritional parameters. 


\section{Conclusions}

Simulations emphasise the large importance of packaging materials and environmental conditions on green beans quality retention during thawing. Materials currently used (e.g. polyethylene bags) provide minimal thermal insulation against temperature abuses. The use of thermal isolating packaging materials provides more time before the complete defrost of the green beans bulk. Model shows that a good insulation material is capable of making a significant decrease on the effect of temperature abuses, by increasing thawing times up to $190 \%$.

Thawing at high temperatures maximises nutritional retention, but sensory retention is compromised. The use of the (high temperatures-short times) HTST principle to frozen green beans thawing is constrained by quality parameters with high activation energies. Thawing inside the refrigerator produces better retentions in terms of colour and flavour, when compared to environmental temperature conditions. Higher quality retentions on the quality profile are obtained by thawing near the melting point temperature, without compromising nutritional or microbiological safety.

Thawing inside the refrigerator is, therefore, recommended for green beans applications that do not need post-processing.

\section{Acknowledgements}

The author R.C. Martins, 2004, gratefully acknowledges his Ph.D. grant, with reference PRAXIS XXI BD/ 18541/98, to the Fundação para a Ciência e Tecnologia (FCT).

\section{References}

Barton, J., \& Nackman, L. (1991). Scientific and engineering C++: an introduction with advanced techniques and examples (5th ed.). Reading, MA: Addison-Wesley.

Bates, D., \& Watts, D. (1988). Non-linear regression analysis and it's applications. New York: Wiley.

Beringer, D. (1997). Modelling global behaviour with scenarios in object oriented analysis. Ph.D. Thesis. Laboratoire du Genie Logiciel, LGL-DI-EPFL, Lausanne, Suisse. Available <http://iglwww.epfl.ch/team/DBR/beringer-thesis_ps.ps>.

Box, G., Hunter, W., \& Hunter, J. (1978). Statistics for experimenters. New York: Wiley.

Byers, T., \& Perry, G. (1992). Dietary carotenes, vitamin C and vitamin E as protective antioxidants in human cancers. Annual Reviews in Nutrition, 12, 139-159.

Dietrich, W. C., Bohart, G. S., Neumann, H. J., \& Morris, H. J. (1959). Time temperature tolerance of frozen foods, xxi: quality retention of frozen green snap beans in retail packages. Food Technology, 13, 136-145.

Dietrich, W. C., Boogs, M. M., Nutting, M. D., \& Weinstein, N. E. (1960). Time temperature tolerance of frozen foods, xxiv: quality changes in cauliflower. Food Technology, 14, 123-128.
Dietrich, W. C., Mildred, M. B., Nutting, M. D., \& Weinstein, N. E. (1960). Time temperature tolerance of frozen foods, xxiii: quality changes in frozen spinach. Food Technology, 14, 522-527.

Favell, D. J. (1998). A comparison of the vitamin C content of fresh and frozen vegetables. Food Chemistry, 62, 59-64.

Fennema, O., Powrie, W., \& Marth, E. (1973). Low-temperature preservation of foods and living matter. New York: Marcel Dekker, Inc.

Gardiner, C. (1997). Handbook of stochastic methods for physics, chemistry and the natural sciences. New York: Springer-Verlag.

Geist, G., Beguelin, A., Dongarra, J., Jiang, W., Manchek, R., \& Sunderam, V. (1994). PVM: parallel virtual machine, a user's guide and tutorial for net worked parallel computing. Cambridge, MA: The MIT Press. Available: http://www.netlib.org/pvm3/book/pvm-book.ps.

Labuza, T. (1982). Shelf-life dating of foods. Wesport, CT: Food \& Nutrition Press.

Martins, R. C. (2004). Modelling temperature abuses to frozen foods and effects on quality. Ph.D. Thesis. Escola Superior de Biotecnologia, Porto, Portugal: Universidade Católica Portuguesa.

Martins, R. C., \& Silva, C. L. M. (2002). Modelling colour and chlorophyll's losses of frozen green beans (Phaseolus vulgaris, L.). International Journal of Refrigeration, 25, 987-995.

Martins, R. C., \& Silva, C. L. M. (2003). Kinetics of frozen stored green beans (Phaseolus vulgaris, L.) quality changes: texture, vitamin C, reducing sugars and starch. Journal of Food Science, 68(7), 2232-2237.

Medeiros, L., Sanik, M., Miller, E., McCombs, K., \& Miller, C. (2001). Performance and microbiological growth in ground meat associated with the use of thawing trays. Journal of Food Quality, 23(4), 409-419.

Meyers, S. (1993). Effective C++. Reading, MA: Addison-Wesley.

Meyers, S. (1998). Effective C++: 50 specific ways to improve your programs and designs. Reading, MA: Addison-Wesley.

Montegomery, D. (1991). Design and analysis of experiments (3rd ed.). Singapore: Wiley.

Neter, J., Kutner, M., Nachtsheine, C., \& Wasserman, W. (1996). Applied linear statistical models (4th ed.). Chicago: Irwin.

Nicolai, B. (1994). Modeling and uncertainty propagation analysis of thermal food processes. Ph.D. Thesis. Leuven, Belgium: Katholieke Universiteit te Leuven, Faculteit Land-bouwkundige en Toegepaste Biologische Wetenschappen.

Pandit, S., \& Wu, S. (1983). Time series and system analysis with applications. Malabar, FL: Krieger Publishing Company.

Priestley, M. (1992). Spectral analysis and time series. London: Academic Press.

Radajewski, J., \& Eadline, D. (1998a). Beowulf how to. Available $<$ http://www.sci.usq.edu.au/staff/jacek/beowulf/beowulf-HOWTO. ps>.

Radajewski, J., \& Eadline, D. (1998b). Beowulf installation and administration how to. Available <http://www.sci.usq.edu.au/staff/jacek/ beowulf/Beowulf-Installation-and-AdministrationHOWTO.ps>.

Ramaswamy, H. S., \& Tung, M. A. (1981). Thermo-physical properties of apples in relation to freezing. Journal of Food Science, 46, 724-728.

Sage, J., \& Ingham, S. (1998). Survival of Escherichia coli 0157:h7 after freezing and thawing in ground beef paties. Journal of Food Protection, 261(9), 1181-1183.

Schwartzberg, H. G. (1976). Effective heat capacities for freezing and thawing of food. Journal of Food Science, 41, 153.

Stat-Ease, (1994). Design-Expert, version 4.0.2. Stat-Ease, Inc.

Sterling, T., Bell, G., \& Kowalik, J. S. (2001). Beowulf cluster computing with Linux. Cambridge: MIT Press.

Tong, H. (1994). Non-linear time series: a dynamical system approach. New York: Oxford University Press.

Yamamoto, S., \& Harris, S. (2001). The effects of freezing and thawing on the survival of Eschericia coli 0157:h7. International Journal of Food Microbiology, 67, 89-96. 